\title{
ESTIMATIVA DOS IMPACTOS ECONÔMICOS DECORRENTES DE EVENTUAL INTRODUÇÃO DO HUANGLONGBING (HLB) NO ESTADO DA BAHIA ${ }^{1}$
}

\author{
JOSÉ MÁRIO CARVALHAL DE OLIVEIRA², ANTÔNIO SOUZA DO NASCIMENTO², \\ SÍLVIA HELENA GALVÃO DE MIRANDA ${ }^{4}$, CRISTIANE DE JESUS BARBOSA ${ }^{5}$, \\ FRANCISCO FERRAZ LARANJEIRAS ${ }^{6}$
}

RESUMO- A Bahia é o segundo mais importante Estado produtor de citros do Brasil, responsável por $5,5 \%$ da produção do País. $80 \%$ da produção vem de propriedades de base familiar, as quais dependem desse cultivo para seu sustento econômico. O Huanglongbing (HLB) nunca foi registrado na Bahia, mas está disseminando-se em outros três Estados do Brasil (São Paulo, Paraná e Minas Gerais), um dos quais faz fronteira com a Bahia. Assim, este estudo teve como objetivo estimar o impacto econômico potencial de uma eventual introdução do HLB na Bahia. Foram utilizados os modelos de Gompertz e logístico associados a diferentes estratos de idade das plantas para estimar, respectivamente, a evolução da incidência e da severidade da doença no parque citrícola baiano, num horizonte de 20 anos. O progresso da doença foi usado para estimar os danos à produção em três cenários. No cenário-base (A), os esforços da agência estadual de defesa agropecuária previniria a entrada do HLB. No cenário B, a doença seria introduzida e não haveria esforços para seu controle, levando a uma disseminação nos anos seguintes. No cenário C, após a deteç̧ão da doença, os produtores adotariam medidas de controle: eliminação de plantas sintomáticas e supressão das populações do inseto vetor. Os custos do controle foram calculados considerando-se a necessidade de pulverizações, inspeções periódicas e eliminação das plantas doentes. O valor presente líquido (VPL) foi usado para comparar os três cenários. Os resultados indicam que as perdas seriam muito significativas caso o HLB fosse introduzido na Bahia. Assim sendo, se as medidas de controle não fossem aplicadas, os prejuízos poderiam superar $\mathrm{R} \$ 1,8$ bilhão.

Termos para indexação: Candidatus Liberibacter, custo, erradicação.

\section{ESTIMATING THE ECONOMIC IMPACT OF AN EVENTUAL INTRODUCTION OF HUANGLONGBING (HLB) IN THE STATE OF BAHIA, BRAZIL}

\begin{abstract}
Bahia is the second most important citrus region in Brazil, accounting for 5,5\% of Brazilian production. $80 \%$ of the production comes from family based farms, which depend on this crop for economic support. Huanglongbing (HLB) was never recorded in Bahia, but is already spreading in three other citrusproducing states of the country, one of which borders the state of Bahia. Thus, this study aimed to estimate the potential economic impact resulting from an eventual introduction of HLB in Bahia. It was used the Gompertz and logistic models to estimate the disease progress under three scenarios. For the baseline scenario (A) the plant health agency efforts would prevent the HLB invasion. In scenario B, we assumed the disease introduction in citrus orchards and the absence of control measures, leading to the spread of HLB in the following years. In scenario C, after detection of the disease, the growers would adopt control measures: eradication of symptomatic hosts and suppression of insect vector populations. The costs of the disease control were calculated by considering the need for insecticide spraying, carrying out periodic inspections and eradication of symptomatic plants. The net present value (NPV) was used for comparing the scenarios. The results showed that, if the HLB is introduced in Bahia, the losses would be very significant in the following 20 years. If control and eradication procedures are not followed, losses in excess of US\$ 890 million could occur.
\end{abstract}

Index terms: Candidatus Liberibacter, cost, eradication.

\footnotetext{
'(Trabalho 002-13). Recebido em: 03-01-2013. Aceito para publicação em: 16-08-2013.

${ }^{2}$ Mestre em Defesa Agropecuária/UFRB. Cruz das Almas-BA. Fiscal Estadual Agropecuário (ADAB). E-mail: Jose.carvalhal@adab.gov.br ${ }^{3}$ Doutor, Pesquisador da EMBRAPA/CNPMF. Prof. do Programa de Pós-Graduação da UFRB. Orientador. E-mail: antnasc@cnpmf. embrapa.br

${ }^{4}$ Doutora, Professora do Departamento de Economia, Administração e Sociologia da ESALQ-USP e pesquisadora do CEPEA. Coorientadora. E-mail: shgdmira@usp.br

${ }^{5}$ Doutora, Pesquisadora da EMBRAPA/CNPMF. Co-orientadora. E-mail: barbosa@cnpmf.embrapa.br

${ }^{6}$ Doutor, Pesquisador da EMBRAPA/CNPMF; E-mail: chico@cnpmf.embrapa.br
} 


\section{INTRODUÇÃO}

Conhecida há mais de um século na China, o huanglongbing (HLB), também chamada de greening, é uma doença devastadora que acomete os citros em nível mundial. Três bactérias gramanegativas, restritas ao floema das plantas infectadas, estão relacionadas a essa doença: Candidatus Liberibacter asiaticus, Candidatus Liberibacter africanus e Candidatus Liberibacter americanus. Destas, apenas a forma asiática e a americana estão presentes no Brasil, com maior predominância da asiática (BOVÉ, 2006). Tais bactérias podem ser transmitidas por material vegetal infectado ou por psilídeos infectivos: Trioza erytreae na África, e Diaphorina citri (Hemiptera: Liviidae) no resto do mundo (SECHLER et al., 2009; BURCKHARDT; OUVRARD, 2012).

No Brasil, o primeiro relato de HLB ocorreu em junho de 2004, em plantas localizadas próximas ao município de Araraquara, São Paulo (COLLETA-FILHO et al., 2004; TEIXEIRA et al., 2005). Após essa detecção, a doença passou a ser considerada o principal problema fitossanitário dos citros do País. Além de São Paulo, o HLB já foi registrado nos Estados do Paraná e Minas Gerais (MAPA, 2009).

Por ainda não haver métodos curativos para o HLB nem para variedades comerciais com algum nível de resistência, essa doença tem causado enormes prejuízos econômicos e sociais, com impactos negativos à citricultura nacional. Segundo Bassanezi et al. (2010a), as epidemias de HLB são rápidas, tendo como consequência imediata a redução na produção, principalmente pela queda precoce de frutos em ramos sintomáticos. Estimativas para o Estado de São Paulo revelaram que a incidência do HLB pode atingir mais de $50 \%$ das plantas em apenas três a cinco anos após o plantio, em propriedades próximas a fontes de inóculo, onde não se realiza o controle do $D$. citri e a supressão das plantas sintomáticas (BASSANEZI et al., 2010a). De acordo com (Bassanezi (2011), de 2005, ano em que teve início o controle oficial do HLB por meio da erradicação de plantas infectadas, até julho de 2011, só no Estado de São Paulo, mais de 12 milhões de árvores de laranja foram erradicadas com sintomas da doença.

Por vir disseminando-se para outras regiões, o HLB constitui uma grande ameaça também à citricultura baiana. A Bahia situa-se em segundo lugar no ranking nacional dos estados produtores de laranja, respondendo por 5,5\% da produção brasileira (IBGE, 2010). A citricultura também é responsável pelo incremento de outras atividades econômicas tanto a montante como a jusante da propriedade rural, como a produção e comercialização de insumos, transporte, distribuição e comercialização da produção e agroindústrias (BAHIA, 2010). Essa atividade reveste-se de maior importância tendo em vista que mais de $80 \%$ da produção baiana é oriunda de produtores enquadrados na chamada agricultura familiar. Esses produtores dependem dessa cultura para sua sustentação econômica e tem área cultivada em média de 10 hectares. Belasque Júnior et al. (2010) demonstraram que o controle do HLB é muito mais difícil em pequenas propriedades que em grandes propriedades na ausência de medidas que promovam o manejo regional da doença (controle do vetor e eliminação de plantas doentes em grandes extensões de área).

Nesse sentido, estimar os impactos econômicos decorrentes da introdução e disseminação do HLB na Bahia é de grande valia, na medida em que permite antever os prejuízos que podem ocasionar à atividade citrícola. Idealmente, tal estimativa permitirá projetar também os recursos que serão necessários para a implementação das ações de defesa sanitária, tanto as preventivas quanto, eventualmente, as de controle. Como o Estado da Bahia é reconhecido pelo Ministério da Agricultura, Pecuária e Abastecimento como área livre do HLB, o presente estudo utilizou informações sobre a evolução da doença e seus danos provenientes do Estado de São Paulo e de outros países onde a doença está presente.

\section{MATERIAL E MÉTODOS}

Adotou-se o pressuposto de que a mesma taxa de crescimento observada para a área plantada com citros na Bahia, entre 2001 e 2010, seria mantida ao longo de 20 anos, até 2031. Assim, a taxa de crescimento geométrico foi obtida por meio do método de regressão linear e foi utilizada para projetar a área plantada com laranja na Bahia entre os anos de 2011 e 2031.

$\mathrm{O}$ estoque de pés de laranja foi calculado por meio da diferença da área plantada no ano pela área plantada no ano anterior. Utilizou-se, para tanto, dos dados disponibilizados pelo IBGE para o período de 1990 a 2010 e o projetado para os anos de 2011 a 2031. Tomou-se como referência a área plantada em 2010, retroagindo-se a 1990, o que permitiu uma aproximação do número de plantas por idade, em cada período, tendo como base o incremento no número de árvores ano a ano. Os dados resultantes são apresentados na Tabela 1 . 
As projeções de incidência e severidade da doença foram feitas por categoria de idade, tendo em vista que o HLB progride e causa prejuízos em graus variáveis de acordo com a idade das plantas (MIRANDA et. al., 2010). Para a projeção da incidência, foi utilizado o modelo de Gompertz, proposto por Bassanezi e Bassanezi (2008) como representativo do progresso da doença no Estado de São Paulo. Tendo em vista que o aumento da incidência é mais rápido em plantas mais jovens, adotaram-se diferentes taxas de progresso para cada categoria de idade: 1,300 para as plantas de 0 a 2 anos; 0,650 (3 a 5 anos); 0,325 (6 a 10 anos) e 0,244 ( $>10$ anos). Considerou-se como incidência inicial o inverso do número de plantas da categoria, tendo em vista que a doença não está presente na Bahia. Ou seja, $1 / \mathrm{n}$, sendo $\mathbf{n}$ o número de pés de laranja de certa idade.

A projeção da severidade por planta foi feita por meio do modelo logístico proposto por Bassanezi e Bassanezi (2008). Esse modelo calcula a severidade num dado tempo como função de dois parâmetros: a taxa de progresso da severidade e a severidade inicial. Para ambos os parâmetros, foram utilizados os mesmos valores propostos por Bassanezi e Bassanezi (2008).

De posse dos dados de severidade para o horizonte de 20 anos, e considerando as diferentes faixas de idade das plantas, pode-se estimar a perda de produtividade das plantas doentes em relação a uma planta saudável de acordo com o modelo proposto por Bassanezi et al. (2011). Essa mesma sequência foi empregada por Miranda et al. (2010, 2011) para projetar os impactos do HLB em diferentes cenários para São Paulo.

$\mathrm{Na}$ etapa seguinte, foi realizada a estimativa dos principais fatores de produção utilizados na citricultura baiana, de modo a projetar os fluxos financeiros do setor em termos de custos e dos impactos da doença sobre os mesmos. O número de tratores envolvidos na citricultura baiana e a terra foram utilizados na estimativa dos fatores de produção, já que representam o estoque de capital na produção agrícola. Para estimativa do estoque de tratores empregados, consideraram-se a projeção da área plantada e o índice de mecanização proposto para a citricultura da Bahia, de um trator para cada 45 ha. Conjecturou-se que o índice de tratores por hectare seria constante ao longo dos 20 anos.

Pela falta de dados referentes ao tempo de utilização de tratores no Brasil, e tendo em vista que eles, depois de esgotada sua vida útil, continuam sendo utilizados nas lavouras, considerou-se como medida de estoque o número total de tratores. A depreciação foi calculada utilizando-se das tabelas de vida útil (anos e horas) e de valor residual elaboradas pela Companhia Nacional de Abastecimento Conab. Para o cálculo da depreciação de máquinas e implementos, foi utilizada a fórmula: Depreciação $=\{[\mathrm{VN}-\mathrm{VR}) / \mathrm{VUa}]$. TOcup $\} /$ área (1), sendo VN o valor do bem novo, VR o valor residual do bem, VUa a vida útil do bem definida em anos e TOcup a taxa de ocupação do bem, definida como o percentual de utilização do bem em uma determinada lavoura, obtido a partir da média de utilização dos tratores nessa lavoura.

A partir da projeção da área plantada, estabeleceu-se o valor do arrendamento da terra para laranja, considerando os diferentes polos de produção da Bahia. Tendo em vista que o objetivo é utilizar o valor dos serviços prestados pelo estoque de capital, a melhor medida a ser utilizada no caso da terra é o arrendamento. Assim, estabeleceu-se como valor do arrendamento o valor obtido por meio da aplicação do percentual de $4 \%$ sobre o valor médio da terra ocupada com laranja no Estado da Bahia (AGRIANUAL, 2010).

Para estimativa do custo operacional, partiuse dos coeficientes técnicos da citricultura baiana elaborado pela Embrapa Mandioca e Fruticultura (2008). Esses coeficientes foram comparados com os indicadores técnicos e econômicos do sistema de produção adotado pelos produtores no principal polo produtor de citros do Estado.

A receita da citricultura foi calculada com base na produção em toneladas de laranja. A partir da área projetada para o Estado, foi estimada a quantidade em toneladas de laranja por ano, considerando-se a produtividade média atual de 20 toneladas por hectare. Posteriormente, estimaramse todos os custos e todas as receitas para o período em estudo, de modo a construir o fluxo de caixa da atividade. O Valor Presente Líquido (VPL) foi calculado então pela seguinte fórmula:

$$
V P L=-P+B A \quad\left[\frac{(1+J)^{n}-1}{J(1+J)^{n}}\right]-C A\left[\frac{(1+J)^{n}-1}{J(1+J)^{n}}\right]+\frac{V R}{(1+J)^{n}}
$$

Em que: P é o valor do investimento inicial (no ano zero);

$$
\left[\frac{(1+J)^{n}-1}{J(1+J)^{n}}\right] \text { é o fator de valor atual, BA é }
$$
o valor anual dos benefícios; CA é o valor anual dos custos; VR é o valor residual futuro dos equipamentos; j é a taxa de juros (taxa de desconto); $n$ é o tempo de maturidade do projeto, que neste caso é de 20 anos. 
Para trazer o fluxo de caixa da citricultura baiana para valores presentes, foram adotadas três taxas, a saber: a da rentabilidade da poupança, a da remuneração do fundo de renda fixa DI e a taxa Selic, todas referentes ao ano de 2010 e divulgadas pelo Banco Central do Brasil.

Todos os cálculos adotaram os seguintes pressupostos: a) consideraram-se apenas as áreas plantadas com laranjeiras. Os demais citros não foram incluídos por não representarem parcela significativa da produção baiana; b) assumiu-se que não haverá solução genética (resistência varietal) para o controle do HLB no período projetado do estudo; c) para iniciar as projeções, assumiu-se que a bactéria do HLB seria introduzida na Bahia no ano de 2012; d) a utilização de insumos e técnicas modernas não aumentaria a produtividade nas lavouras ; e) os valores utilizados no estudo, como a receita com os citros e os custos, foram corrigidos para os 20 anos de projeção, tomando por base uma inflação anual de $4,5 \%$, baseada nas metas de inflação do Banco Central do Brasil divulgada para o ano de 2011 ; f) tendo em vista que os custos relativos à detecção e ao controle do HLB variam consideravelmente, eles foram atualizados utilizando a taxa de câmbio média real de US\$ 1,67, disponibilizada pelo Banco Central do Brasil para o ano de 2010; g) os eventuais impactos ambientais, pelo possível aumento do uso de agrotóxicos, não foram valorados; h) pressupõe-se, que a partir da adoção dos métodos de controle do HLB, a produtividade dos pomares seria mantida nos níveis atuais ao longo do horizonte de projeção; i) conjecturou-se que o preço da tonelada de laranja não sofreria alteração durante o período do estudo. Portanto, utilizou-se o valor médio da tonelada de laranja para o Estado da Bahia (IBGE, 2010), corrigido para os anos de 2011 a 2031, utilizandose da meta de inflação, conforme já mencionado acima.

Finalmente, três cenários de disseminação do HLB foram comparados pelo VPL. No cenário A, considerou-se que os esforços da defesa sanitária impediriam a introdução da bactéria causadora do HLB nos pomares de citros, nos diferentes polos produtores da Bahia (cenário base). No cenário B, assumiu-se a introdução da bactéria e a não adoção, pelos produtores, das medidas preconizadas para controle da doença. Haveria, portanto, disseminação nos anos seguintes (cenário com as estimativas de perdas máximas). Neste cenário, o progresso da incidência da doença e da severidade nos pomares foi o mesmo utilizado por Miranda et al. (2011) para avaliar os custos e benefícios envolvidos nas ações dos governos e do Fundecítrus no controle do HLB em São Paulo. No cenário $C$, após a detecção da doença em 2012, os produtores, em parceria com a Agência Estadual de Defesa Agropecuária da Bahia $(\mathrm{ADAB})$ e a Empresa Baiana de Desenvolvimento Agrícola (EBDA), implementariam as medidas de controle e a erradicação recomendadas para a doença. Essas medidas teriam como objetivos: (i) reduzir as fontes de inóculo, por meio da eliminação de plantas doentes (ou roguing) imediatamente após a detecção, e (ii) reduzir a probabilidade de ocorrência de novas infecções, por meio do controle químico do inseto vetor. Assim, o cenário $C$ não implica, portanto, a erradicação total da doença. Os custos de controle da doença foram medidos por meio da necessidade de pulverizações, da realização das inspeções periódicas e da erradicação das plantas com sintomas.

\section{RESULTADOS E DISCUSSÃO}

As projeções indicam que, no cenário B (presença do HLB sem controle), a redução na produção começaria no terceiro ano após a introdução do HLB no Estado, atingindo 19\% após seis anos e $60 \%$ no nono ano após a contaminação dos pomares (Gráfico 1). No cenário $\mathrm{C}$ (presença do HLB com controle), em que pesem os custos inerentes às atividades de monitoramento e controle, a redução na produção de laranja seria inferior a $1 \%$ para todos os anos estudados.

A diferença nos valores líquidos presentes das margens brutas dos três cenários indica a possibilidade de significativo impacto econômico do HLB no Estado da Bahia (Tabela 2). Tomando como base a rentabilidade da poupança $(11,71 \%$ ao ano), no cenário base (A), no qual se considerou que os esforços da defesa sanitária seriam efetivos em excluir a bactéria causadora do HLB por todo o período estudado, o VPL da margem bruta foi positivo, atingindo $\mathrm{R} \$ 1,520$ bilhão. No cenário B (introdução do HLB e ausência de controle), o VPL da margem bruta foi negativo, alcançando R\$ -316 milhões, indicando prejuízo financeiro para os produtores. Já no cenário $\mathrm{C}$ (detecção da doença com implementação de medidas de controle e erradicação), o VPL da margem bruta foi de R\$ 685 milhões.

Comparando os cenários A e $\mathrm{B}$, o prejuízo líquido seria de $\mathrm{R} \$ 1,837$ bilhão (Tabela 2). Ao deixar de controlar a doença caso ela seja introduzida na Bahia, os produtores, além de perderem $\mathrm{R} \$ 316$ milhões em custos acima de suas receitas, deixariam de ganhar R\$ 1,520 bilhão. Estudos realizados 
para o Estado de São Paulo, em um cenário sem controle do HLB, indicaram que as perdas totais alcançariam R \$ 25,4 bilhões para um horizonte de 20 anos, utilizando-se da TJLP (MIRANDA et al. 2011). Obviamente tal diferença deve ser atribuída à magnitude das citriculturas nesses estados, e não a um comportamento diferente da doença.

$\mathrm{Se}$ as medidas de controle fossem implementadas após a detecção da doença, o benefício líquido de seu controle totalizaria $\mathrm{R} \$$ 1,002 bilhão (Tabela 2). Neste caso, os produtores não perderiam os $\mathrm{R} \$ 316$ milhões do cenário $\mathrm{B}$ e ganhariam R\$ 685 milhões, em valor presente líquido durante os 20 anos. No Estado de São Paulo, em um cenário com controle do HLB, as perdas totais alcançariam R $\$ 4$ bilhões para um horizonte de 20 anos, utilizando-se da TJLP (MIRANDA et al., 2011).

Cumpre mencionar que, na ilustração dos impactos acima, destacam-se os valores obtidos, utilizando-se da taxa da poupança e da TJLP, no caso do estudo referenciado. Essas taxas, em geral, são mais baixas, e sua opção como taxa de desconto pode evidenciar a preocupação com uma taxa que reflita aspectos sociais relevantes do projeto que não são mensurados apropriadamente em sua análise. Isto é particularmente importante quando se pensa na avaliação de políticas de defesa fitossanitária como um investimento.

Finalmente, comparando-se o VPL do cenário A com o cenário C (Tabela 2), os produtores de citros deixariam de ganhar $\mathrm{R} \$ 835$ milhões devido à entrada da doença no Estado. Isso ocorreria mesmo considerando que o Estado e a iniciativa privada investiriam para evitar sua disseminação (Tabela 2).

A estimativa de R \$ 1, 837 bilhão em prejuízos potenciais líquidos diretos justifica os esforços dos órgãos responsáveis pela defesa sanitária em impedir a entrada da doença. Tais esforços são claramente menos onerosos do que seu controle após a introdução. Entretanto, considerando que na Bahia a maior parte dos produtores de laranja é de origem familiar, é certo que o impacto vá além daquele montante, em função da provável dispensa da mão de obra envolvida na atividade. Além disso, haveria efeitos a montante e a jusante da propriedade rural em decorrência da disseminação da doença. Especificamente, seriam afetados setores como a produção e a comercialização de insumos e serviços, a distribuição, o processamento e a comercialização de laranjas, além da arrecadação de impostos.

É importante destacar que a estimativa do impacto social não foi realizada no presente estudo. Há um alto grau de informalidade da atividade no Estado, tendo em vista que $80 \%$ dos agricultores envolvidos são considerados agricultores familiares. Mais ainda, em sua grande maioria, eles não têm acesso aos programas de crédito e assistência técnica. Assim, a estimativa do impacto social vis a vis à introdução do HLB é matéria de interesse a ser examinada em estudo futuro.

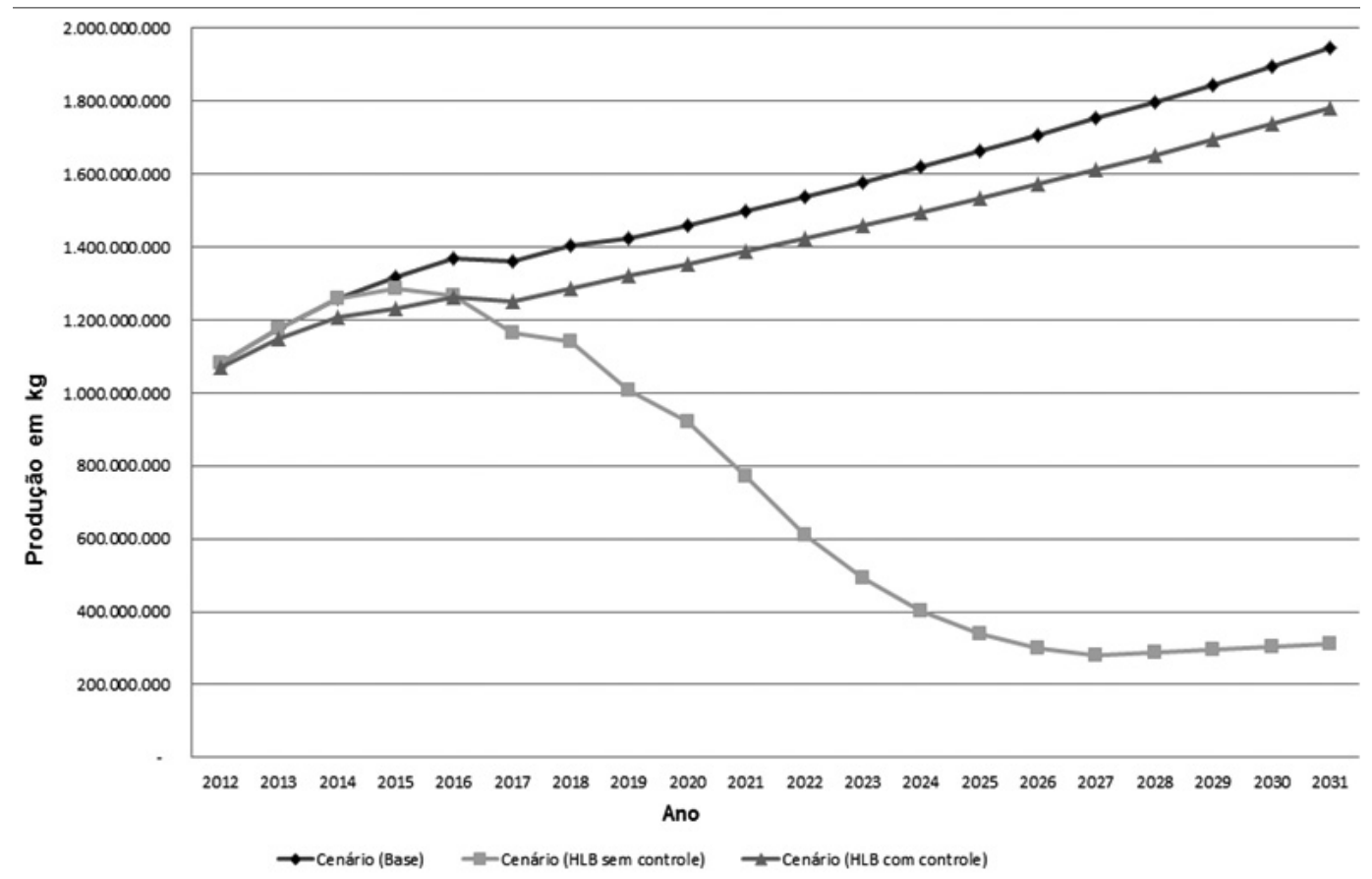

FIGURA 1 - Produção de laranja nos cenários estudados para o período de 2012 a 2031. Bahia, em kg. 2012. 
TABELA 1 - Estimativa do número de árvores de laranja por categoria no Estado da Bahia de 2011 a 2031.

\begin{tabular}{lccccc}
\hline Ano & Total de Árvores & 0 a 2 anos & 3 a 5 anos & 6 a 10 anos & $>10$ anos \\
\hline 2011 & 26.109 .120 & 2.915 .040 & 4.605 .952 & 2.701 .088 & 15.887 .040 \\
2012 & 26.798 .401 & 1.360 .833 & 6.560 .736 & 2.458 .144 & 16.418 .688 \\
2013 & 27.505 .878 & 1.396 .759 & 2.915 .040 & 6.775 .392 & 16.418 .688 \\
2014 & 28.232 .034 & 1.433 .633 & 3.604 .321 & 5.930 .080 & 17.264 .000 \\
2015 & 28.977 .359 & 1.471 .481 & 2.068 .310 & 8.030 .464 & 17.407 .104 \\
2016 & 29.742 .361 & 1.510 .328 & 2.122 .914 & 7.520 .992 & 18.588 .128 \\
2017 & 30.527 .560 & 1.550 .201 & 2.178 .959 & 7.921 .569 & 18.876 .832 \\
2018 & 31.333 .487 & 1.591 .126 & 2.236 .483 & 4.311 .798 & 23.194 .080 \\
2019 & 32.160 .691 & 1.633 .132 & 2.295 .526 & 5.037 .954 & 23.194 .080 \\
2020 & 33.009 .734 & 1.676 .246 & 2.356 .128 & 3.539 .791 & 25.437 .568 \\
2021 & 33.881 .191 & 1.720 .499 & 2.418 .330 & 3.633 .242 & 26.109 .120 \\
2022 & 34.775 .654 & 1.765 .920 & 2.482 .174 & 3.729 .159 & 26.798 .401 \\
2023 & 35.693 .731 & 1.812 .541 & 2.547 .703 & 3.827 .609 & 27.505 .878 \\
2024 & 36.636 .046 & 1.860 .392 & 2.614 .963 & 3.928 .658 & 28.232 .034 \\
2025 & 37.603 .238 & 1.909 .506 & 2.683 .998 & 4.032 .375 & 28.977 .359 \\
2026 & 38.595 .963 & 1.959 .917 & 2.754 .855 & 4.138 .829 & 29.742 .361 \\
2027 & 39.614 .896 & 2.011 .659 & 2.827 .583 & 4.248 .094 & 30.527 .560 \\
2028 & 40.660 .730 & 2.064 .767 & 2.902 .232 & 4.360 .244 & 31.333 .487 \\
2029 & 41.734 .173 & 2.119 .277 & 2.978 .851 & 4.475 .354 & 32.160 .691 \\
2030 & 42.835 .955 & 2.175 .225 & 3.057 .492 & 4.593 .504 & 33.009 .734 \\
2031 & 43.966 .824 & 2.232 .651 & 3.138 .210 & 4.714 .772 & 33.881 .191 \\
\hline & & & & &
\end{tabular}

TABELA 2 - Valor Presente Líquido (VPL) em reais da margem bruta na citricultura baiana para diferentes cenários e taxas de desconto. Projeção 2011-2031.

\begin{tabular}{c|c|c|c|c|c|c|}
\hline $\begin{array}{c}\text { Taxa de } \\
\text { desconto \% }\end{array}$ & $\begin{array}{c}\text { VPL Cenário A } \\
\text { (ausência do HLB) } \\
\text { RS }\end{array}$ & $\begin{array}{c}\text { VPL Cenário B } \\
\text { (HLB sem controle) } \\
\text { RS }\end{array}$ & $\begin{array}{c}\text { VPL Cenário C } \\
\text { (HLB com controle) } \\
\text { RS }\end{array}$ & $\begin{array}{c}\text { Diferença } \\
\text { Cenário A-B } \\
\text { RS }\end{array}$ & $\begin{array}{c}\text { Diferença } \\
\text { Cenário C-B } \\
\text { RS }\end{array}$ & $\begin{array}{c}\text { Diferença } \\
\text { Cenário A-C } \\
\text { RS }\end{array}$ \\
\hline 11,71 & 1.520 .603 .129 & -316.951 .160 & 685.197 .751 & 1.837 .554 .289 & 1.002 .148 .911 & 835.405 .378 \\
\hline 15 & 1.180 .570 .240 & -55.594 .603 & 535.767 .329 & 1.236 .164 .843 & 591.361 .932 & 644.802 .911 \\
\hline 15,64 & 1.128 .054 .558 & -19.610 .656 & 512.521 .050 & 1.147 .665 .214 & 532.131 .706 & 615.533 .508 \\
\hline
\end{tabular}

Nota: No cenário (A) as ações da agência estadual de defesa agropecuária previniria a entrada do HLB no Estado. No cenário B, a doença seria introduzida e não haveria esforços para seu controle. No cenário C, após a detecção da doença, os produtores adotariam medidas de controle. 


\section{CONCLUSÃO}

1-Assumindo-se que o HLB será introduzido no Estado da Bahia, as perdas econômicas potenciais para a citricultura baiana serão bastante elevadas; sobretudo se for considerada uma eventual falta de ações governamentais e privadas para o controle de sua disseminação no Estado. Nesse cenário, os agricultores, por não estarem capacitados para reconhecerem os sintomas da doença ou por estarem descapitalizados, não adotariam o manejo preconizado. Outra justificativa para que o cenário de dispersão da doença na Bahia ocorra, seria uma falta de ação dos órgãos competentes ou uma ação insuficiente. No Estado de São Paulo, após a mudança na legislação sobre inspeção e controle do HLB, que reduziu a ação dos órgãos encarregados pela defesa sanitária em 2009, a incidência cresceu bastante (MAPA, 2009).

2-Assim, o controle rígido do trânsito de vegetais é absolutamente necessário, sobretudo quando oriundo de regiões/Estados onde o HLB está presente. Além disso, a utilização de mudas oriundas de viveiros em ambiente telado, ações de educação sanitária e o monitoramento do inseto vetor do HLB seriam medidas vitais para retardar a introdução e o estabelecimento da doença na Bahia ou em qualquer outra região onde ela ainda não foi relatada.

\section{REFERÊNCIAS}

AGRIANUAL: anuário da agricultura brasileira. São Paulo: FNP, Consultoria e Agroinformação, $2010.520 \mathrm{p}$.

BAHIA (Estado). Secretaria da Agricultura, Irrigação e Reforma Agrária. Programa de desenvolvimento sustentável da citricultura baiana. Salvador, 2010. $20 \mathrm{p}$.

BASSANEZI, R.B.; BASSANEZI, R.B An approach to model the impact of Huanglongbing on citrus yield. In: INTERNATIONAL RESERACH CONFERENCE ON HUANGLON BING, 2008, Orlando, Proceedings... p.301-304. Disponível em: $<$ www.plantmannagementnetwork.org $>$. Acesso em: dec. 2008.
BASSANEZI, R. B.; LOPES, S. A.; BELASQUE Jr.; J.; SPÓSITO, M. B.; YAMAMOTO, P. T.; MIRANDA, M. P.; TEIXEIRA, D. C.; WULFF, N. A. Epidemiologia do Huanglongbing e suas implicações para o manejo da doença. Citrus Research \& Tecnology, Cordeirópolis, v.31, n.1, p 11-23, 2010a.

BASSANEZI, R. B.; MONTESINO, L. H.; GASPAROTO, M. C. G.; BERGAMIN FILHO, A.; AMORIM, L. Yield loss caused by huanglongbing in different sweet Orange cultivars in São Paulo, Brazil. European Journal of Plant Pathology, Dordrecht, v.130, n.4, p.577-586, 2011.

BASSANEZI, R. B. Ameaça iminente à citricultura do Nordeste Brasileiro. Salvador: Embrapa/MAPA/ ADAB/EBDA. 2011. Disponível em: $<$ http://www. cnpmf.embrapa.br/publicacoes/Anais HLB/anais encontro_HLB/Palestra_Renato_Bassanezi.pdf $>$. Acesso em: 2011.

BELASQUE JUNIOR , J. ; BASSANEZI, R.B.; YAMAMOTO, P.T.; AYRES, A. J.; TACHIBANA, A.; VIOLANTE, A.R.; TANK JUNIOR, A.; DI GIORGI, F.; TERSI, F.E.A.; MENEZES, G.M.; DRAGONE, JANK JUNIOR, J.; R.H.; BOVÉ, J.M. Lessons from huanglongbing management in São Paulo state, Brazil. Journal of Plant Pathology, Wageningen, v.92, n.2, p. 285-302, 2010.

BOVÉ, J. M. Huanglongbing: a destructive, newlyemerging, century-old disease of citrus. Journal of Plant Pathology, Wageningen, v.88, p.7-37, 2006.

BURCKHARDT, D.; OUVRARD D. A revised classification of the jumping plant-lice (Hemiptera: Psylloidea). Zootaxa, New Zealand, n.3509, p.134, 2012.

COLLETA-FILHO, H.D.; TARGON, M.L.P.N.; TAKITA, M.A.; DE NEGRI, J.D; POMPEU JR, J.; MACHADO, M.A.; DO AMARAL, A.M.; MULLER, G.W. First Report of the Causal Agent of Huanglongbing (Candidatus Liberibacter asiaticus) in Brazil. Plant Disease, Saint Paul, v. 88, n. 12, p. 1382, 2004.

IBGE - Instituto Brasileiro de Geografia e Estatística. 2010. Disponível em : <http:/Iwww. IBGE.gov.br/sidra $>$. Acesso em: 15 mar. 2011. 
MAPA. Ministério da Agricultura, Pecuária e Abastecimento. Manual de procedimentosHuanglongbing: levantamentos fitossanitários, ações de prevenção e de controle. Brasília. 2009. 6p.

MIRANDA, S. H. G; BASSANESI, R. B; ADAMI, A. C. O. ABC das ações de defesa fitossanitária para o HLB em São Paulo: lições para a Citricultura do Nordeste. Revista Bahia Agrícola, Salvador, v.9, p.64-71, 2011.

MIRANDA, S.H.G.; NASCIMENTO, A.M.; XIMENES, V.; BASSANEZI, R. B. Uma aplicação da análise benefício-custo para políticas de defesa sanitária: alguns estudos de caso para o Brasil. Brasília: CNPq, 2010. 100 p. (Relatório de Pesquisa)
SECHLER, A.; SCHUENZEL E. L.; COOKE, P.; DONNUA, S.; THAVEECHAI, N.; POSTINIKOVA, E.; STONE, A. L.;SCHNEIDER, W. L.; DAMSTEEGT, V. D.; SCHAAD, N. W. Cultivation of 'Candidatus Liberibacter asiaticus', ' Ca. L. africanus' and 'Ca. L. americanus' Associated with Huanglongbing. Phytopathology, St. Paul, v. 99, p. 480-486, 2009.

TEIXEIRA, D.C; SAILLARD, C.; EVEILLARD, S.; DANET, J.L; COSTA, P.I.; AYRES, A.J.; BOVE, J. 'Candidatus Liberibacter americanus', associated with citrus Huanglongbing (greening disease) in São Paulo state, Brazil. International Journal of Systematic and Evolutionary Microbiology, Reading, v. 55, p. 1857-1862, 2005. 